H I G H L I G H T S

\section{CELL CYCLE}

Divide and rule

As centrosome duplication and separation are linked to the cell cycle, and as cyclin-dependent kinases (CDKs) are crucial for cell-cycle progression, researchers believe that centrosome duplication is linked to the activation of CDKs. Two cyclin-CDK complexes, cyclin E-CDK2 and cyclin A-CDK2, have been implicated in the centrosome cell cycle, and several CDK2 substrates have been identified. Brian Dynlacht and colleagues now report in Developmental Cell the identification of a new $\mathrm{CDK}$ substrate with a function in controlling the centrosome cell cycle.

With the goal of identifying new CDK targets, Dynlacht and co-workers screened a human cDNA expression library with a radiolabelled, dominantnegative form of cyclin E-CDK2. Among the positive clones were two known cyclin-CDK interaction partners, which indicated that the screen had been successful. Dynlacht and colleagues chose to study another clone that encoded a $110-\mathrm{kDa}$, previously uncharacterized protein - a protein they call 'centrosomal protein of 110 $\mathrm{kDa}$ or CP110. This protein does not resemble any known proteins, but among its recognizable motifs are two cyclin-binding domains and ten putative $\mathrm{CDK}$ phosphorylation sites.

So, is CP110 a CDK substrate? GST-tagged CP110 was phosphorylated in vitro by cyclin $\mathrm{E}-\mathrm{CDK} 2$, cyclin A-CDK2 and also by cyclin B-CDC2, which are all active from the $S$ phase and through the M phase - but not by a G1-phase kinase. CP110 was also phosphorylated in vivo, and the pattern of phosphopeptides following tryptic digestion resembled that of the in vitro phosphorylations. This indicates that CP110 is an authentic in vivo substrate for cyclin-CDK complexes.

Using northern and western blot analysis of extracts prepared from synchronized cells, Dynlacht and colleagues showed that $\mathrm{CP} 110$ expression increased as cells progressed through the G1-to-S-phase transition, and peaked during $S$ phase, after which
CP110 levels diminished. So, the expression of $\mathrm{CP} 110$ coincides with the activation of CDK2 and centrosome duplication and separation.

Next, Dynlacht and colleagues determined the subcellular localization of CP110 by immunofluorescence, and found that $\mathrm{CP} 110$ antibody staining overlaps with two centrosomal markers, $\gamma$-tubulin and centrin. They confirmed the centrosomal localization of CP110 by taking a biochemical approach - centrosomes that were purified through a sucrose gradient contained CP110.

To test the function of CP110, Dynlacht and co-workers downregulated CP110 levels by RNA interference (RNAi). They then arrested the cells in $S$ phase - an event that would normally provoke centrosome amplification, but which, instead, caused blockage of centrosome duplication. Stable expression of a nonphosphorylated mutant version of CP110 resulted in polyploidy. Equally, depletion of CP110 levels by RNAi or loss of CP110 phosphorylation increased unscheduled centrosome separation.

The authors concluded that CP110 might function in two ways - by positively regulating centrosome duplication and by suppressing premature centrosome separation. The next step will be to elucidate the actual mechanism through which CP110 functions. The finding that polyploidy results from mutating CP110 implies a direct link between centrosome function and genomic stability. CP110 might also reveal new insights into a possible connection between centrosome defects and cancer. So, it will be a priority to investigate whether the $C P 110$ gene is a target of mutations in human cancers. Arianne Heinrichs

\section{(2) References and links} ORIGINAL RESEARCH PAPER Chen, Z. et al. CP110, a cell cycle-dependent CDK substrate, regulates centrosome duplication in human cells. Dev. Cell 3, 339-350 (2002)

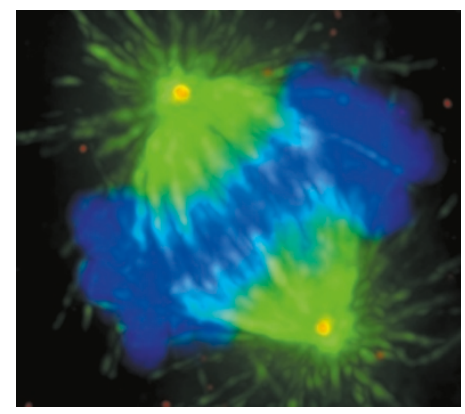

\section{IN BRIEF}

\section{CELLULAR MICROBIOLOGY}

Conversion of Ptdlns $(4,5) \mathrm{P}_{2}$ into Ptdlns(5)P by the $S$. flexneri effector lpgD reorganizes host cell morphology.

Niebuhr, K. et al. EMBO J. 21, 5069-5078 (2002)

The virulence factor IpgD from Shigella flexneri has been implicated in entry focus formation. Niebuhr et al. now show that IpgD acts as a phosphoinositide phosphatase that dephosphorylates phosphatidylinositol 4,5-bisphosphate. The resulting phosphatidylinositol 5-monophosphate causes a decrease in membrane tether force that eventually promotes membrane ruffling at the entry site - and thereby increases the pathogen's virulence.

\section{DEVELOPMENT}

Bicoid associates with the 5'-cap-bound complex of caudal $\mathrm{mRNA}$ and represses translation.

Niessing, D. et al. Genes \& Dev. 16, 2576-2582 (2002)

Pattern formation during early Drosophila embryogenesis is initiated by the asymmetric distribution of several transcription factors, including Bicoid (BCD). BCD causes specific translational repression of caudal (cad) mRNA. Translational repression involves not only the binding of $\mathrm{BCD}$ to a cis-element in the 3 -untranslated region (UTR) of the mRNA, but also depends as Niessing and colleagues now show - on an eIF4E-binding motif in $\mathrm{BCD}$, which is required for cap-dependent translation initiation. The researchers propose that the $3^{\prime}$-UTR-bound BCD interferes with the assembly of the initiation complex.

\section{SIGNALLING}

DAP-kinase induces apoptosis by suppressing integrin activity and disrupting matrix survival signals.

Wang, W.-J. et al. J. Cell Biol. 159,169-179 (2002)

Death-associated protein kinase (DAP-kinase), as its name suggests, has a role in apoptosis, which, until now, has remained unclear. This report provides evidence that DAP-kinase exerts an inhibitory effect on integrin function, which negatively regulates integrin-mediated adhesion. Integrin-extracellular-matrix-mediated survival signals are consequently suppressed, which, through the activation of $\mathrm{p} 53$ dependent pathway, induces anoikis-like apoptosis.

\section{REPLICATION}

De novo formation of centrosomes in vertebrate cells arrested during $\mathrm{S}$ phase.

Khodjakov, A. et al. J. Cell Biol. 158, 1171-1181 (2002)

Centrosomes replicate through a semiconservative mechanism, and their number is controlled by the cell's ploidy. Earlier studies had indicated that centrosomes do not regenerate, and that centrosome assembly requires a specific 'template'. However, Khodjakov and colleagues now show that when centrosomes are destroyed by laser surgery in $\mathrm{CHO}$ cells arrested in $\mathrm{S}$ phase, new centrosomes form de novo. Pericentriolar material appears after $5-8$ hours, and centrioles by 24 hours. 